\title{
Mid-Infrared Imaging of Post-AGB Objects
}

\author{
C. Kömpe $^{1}$, T. Lehmann ${ }^{1}$, J. Gürtler ${ }^{1}$, B. Stecklum ${ }^{2}$ and E. Krügel ${ }^{3}$ \\ ${ }^{1}$ Universitäts-Sternwarte; ${ }^{2}$ Karl-Schwarzschild-Observatorium; \\ ${ }^{3}$ Max-Planck-Institut für Radioastronomie
}

The evolution of low- and intermediate-mass stars (about 1 to $8 M_{\odot}$ ) from the asymptotic giant branch (AGB) to the planetary nebulae stage is characterized by intense stellar wind. AGB stars evolve on a very short time scale (several thousand years) via a phase, where the star is hidden from view by a dense dust shell, towards the post-AGB phase (an optically visible star surrounded by a cold dust/gas shell). The observational investigation of these shells is rather difficult due to their small angular extent. However, the resolution of current infrared telescopes is sufficient to resolve the warm dust of the inner part of the envelope.

In order to study the structure of the warm dust surrounding the post-AGB objects IRAS 07134+1005, IRAS 07399-1435, and IRAS 16342-3814, we have carried out Nband $(10.6 \mu \mathrm{m})$ observations using the ESO $2.2 \mathrm{~m}$ telescope with the new MANIAC infrared camera of the MPE in Garching, Germany (Böker 1996). For the observations, we used a chopping and nodding technique with an on-source integration time of 8 to $16 \mathrm{~min}$. The spatial resolution of the images is about $1^{\prime \prime}$. We determined the point spread function on $\alpha \mathrm{CMa}$ and $\alpha \mathrm{CMi}$.

The comparison of the radial intensity distribution of the sources with the point spread function shows that IRAS $07134+1005$ is clearly resolved while the other two sources are only somewhat more extended than a point source. We have deconvolved the images of all three objects with the point spread function. Nonspherically extended emission with a diameter of about $4^{\prime \prime}$ is seen on all images. Only the image of IRAS $07134+1005$ shows a shell structure. The morphology of this shell is very similar to that reported by Meixner et al. (1994).

We have carried out radiative transfer calculations, assuming a spherical symmetry of the dust shell, in order to model the spectral energy distribution of our objects. From the model results, we have derived a theoretical radial intensity distribution at which was convolved with the point spread function. The comparison of the theoretical with the observed radial distribution showed that the theoretical distribution is systematically narrower than the observed one. This result may indicate that some assumptions made in our model are probably incorrect. The radial intensity distribution must thus be considered an important additional constraint on radiative transfer calculations of circumstellar envelopes.

Acknowledgement. This work has been partially supported by the German BMBF(Förderkennzeichen 05 2JN13A).

\section{REFERENCES}

Böker, T., 1996, PhD thesis, MPI f. Extrater. Physik, Garching, Germany, in prep.

Gürtler, J., Kömpe, C., Henning, Th., 1996, Astron. Astrophys., 305, 878

Meixner et al., 1994, Exp. Astron., 3, 53 